\title{
Ethylene Glycol as Entrainer in 1-Propanol Dehydration: Scrutiny of Physicochemical Properties of Ethylene Glycol+1-Propanol Binary Mixture at Different Temperatures
}

\author{
Sanghamitra Pradhan, Sujata Mishra, Lopamudra Acharya
}

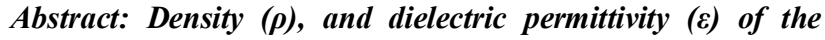
binary mixture of ethylene glycol + 1- propanol have been determined as a function of mole fraction of ethylene glycol at $T$ $=(298,308$ and 313)K. These measurements have been employed to compute thermodynamic properties like molar volume $(V)$, and molar polarization $(P)$ of the liquid mixtures. Excess properties such as $V E, \varepsilon E$ and $P E$ for the binary mixture have also been calculated at different temperatures. In order to calculate the standard deviations of the excess properties, Redlich - Kister polynomial equation was used. The negative and positive values of the excess properties predicted the type and strength of molecular interactions between the components in the binary mixture.The excess molar volume for the mixture at all temperatures is found to be negative. The $\varepsilon E$ values for ethylene glycol + 1-propanol has been found to be negative at $298 \mathrm{~K}$ and but positive values are obtained at 308 and $313 \mathrm{~K}$. The divergent magnitudes of $\varepsilon E$ values at different temperatures have been attributed to different strengths of hydrogen bond interactions as well as alignments of net dipoles.
\end{abstract}

Keywords: Binary mixture, Ethylene glycol, Excess properties, 1-propanol, Temperature.

\section{INTRODUCTION}

Investigations on various thermo physical and thermodynamic properties of binary mixtures on the basis of a wide range of mole fractions and temperatures furnish useful information concerning the type of intermolecular associations [1]. Studies on volumetric properties of solutions help in interpreting various types of solute-solvent interactions. Additional parameters such as apparent molar volumes, limiting apparent molar volumes also serve as important tools for analyzing the various thermodynamic interactions in the chosen binary mixture.

Pal and Singh have reported excess molar volumes and viscosities of different kind of ethylene glycol binary mixtures at 298.15 and $308.15 \mathrm{~K}$ [2]. Investigations on deviation of refractive index, viscosity of mixture and deviation in molar volume for aqueous solution of alcohol and ethylene glycol at $291.15 \mathrm{~K}$ and atmospheric pressure have also been reported [3].Cruz et al. carried out viscosity

Revised Manuscript Received on September 14, 2019. Technical Education and Research, Siksha'O'Anusandhan Deemed to be University, Khandagiri square, Bhubaneswar, Odisha, India.

Sujata Mishra, Department of Chemistry, Institute of Technical Education and Research, Siksha'O'Anusandhan Deemed to be University, Khandagiri square, Bhubaneswar, Odisha, India.(Email: drsujatamishra97@gmail.com/sujatamishra@soa.ac.in)

Lopamudra Acharya, Department of Chemistry, Institute of Technical Education and Research, Siksha'O'Anusandhan Deemed to be University, Khandagiri square, Bhubaneswar, Odisha, India.
Sanghamitra Pradhan, Department of Chemistry, Institute of

studies for the first six members of poly (ethylene glycols) (PEGs) at different temperatures and established a generalized correlation basing on the number of carbon atoms [4]. The physicochemical properties of ethylene glycol and water mixture at various temperatures have been investigated by Yang et al [5]. The negative excess molar volumes over the entire range of composition at low temperatures predicted the existence of specific interactions between unlike molecules.

Dielectric investigations elucidate the intermolecular interactions in the binary mixtures which occur due to dipole-dipole, dipole-induced dipole interactions and hydrogen bonding. Several researchers have identified the homo and hetero interactions in various liquid systems with the help of dielectric measurements [6]. Ray et al. have evaluated the excess Gibb's energy of mixing in the polarnonpolar binary mixture using dielectric probe and proposed an equation to find out excess Gibb's energy [7]. Dielectric permittivity reports of binary mixtures of ethylene glycol mono phenyl ether with alcohols at varied temperatures have been investigated by Rana and Chaube [8]. Dielectric properties of binary mixtures of phenyl ether of ethylene glycol with methanol have also been examined. Rana et al. have studied the physical, electrical and optical behaviour of mixtures of anisole and alcohols at different temperatures [9].

Alcohols serve as an important class of organic compounds which have industrial and scientific significance. These are strongly associated in solution because of dipole-dipole interaction and hydrogen bonding (Figure 1). Increasing attention has recently been paid to find alternative source of energy due to adverse effect of fossil fuels on the environment. One of these proposals is use alcohols as biofuels from renewable energy sources [10]. With the help of esterification process the conversion of 1-propanol to diesel fuel can be carried out [11]. With the azeotropic mixture of 1-propanol and water, the dehydration of 1-propanol becomes very tedious with conventional distillation process. The separation of the mixture becomes feasible with the aid of extractive distillation process by employing a third component in the process to break the azeotrope [12]. Franco et al. have discussed the prospective of ethylene glycol as entrainer in 1- propanol dehydration [13]. 


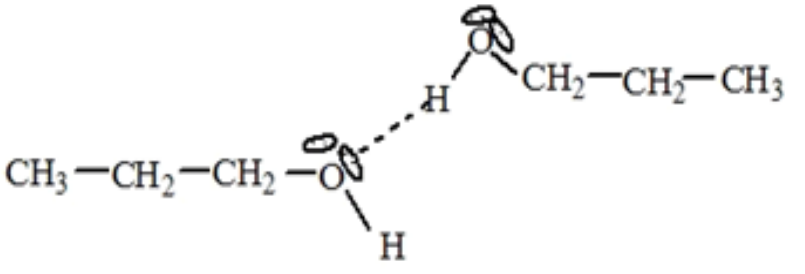

Figure 1: Representation of intermolecular hydrogen bonding in propanol.

Keeping in mind the potential use of ethylene glycol as entrainer, the present investigation focuses on the molecular interactions resulting in modifications of molecular and dipolar orientation between 1-propanol and ethylene glycol at different temperatures $(\mathrm{T}=298,308$ and 313K). Few thermo physical data are available in the literature concerning this system at variable temperatures. The excess volume and dielectric permittivity have been evaluated for clear understanding of the thermodynamics of the pure components as well as mixture. Mixing rules proposed by different researchers for dielectric measurements have been used which are significant from theoretical and applied research point of view.

\section{EXPERIMENTAL}

\section{A. Materials}

Ethylene glycol and 1-propanol were obtained from Merck. The state of purity was greater than 0.99 mass percent. The volume loss of chemicals has been eluded with the use of air tight containers. The assessment of pureness of the chemicals has been done by relating the measured densities and dielectric permittivity given in Table 1 with the available literature and the matching of data was observed to satisfactory level.

Table1-List of experimental and literature values of densities and dielectric permittivity of ethylene glycol and 1- propanol at 298, 308 and $313 K$.

\begin{tabular}{cccc}
\hline Components & $T / K$ & $\begin{array}{c}\rho\left(\mathrm{g} / \mathrm{cm}^{3}\right) \\
(\text { expt })\end{array}$ & $\begin{array}{c}\rho\left(\mathrm{g} / \mathrm{cm}^{3}\right) \\
(\text { lit })\end{array}$ \\
\hline $\begin{array}{ccc}\text { Ethylene glycol } \\
\left(X_{2}\right)\end{array}$ & $298 \mathrm{~K}$ & 1.10500 & $1.10950[13]$ \\
& $308 \mathrm{~K}$ & 1.10200 & $1.10250[14]$ \\
& $313 \mathrm{~K}$ & 1.09800 & $1.09835[14]$ \\
1-propanol $\left(X_{1}\right)$ & $298 \mathrm{~K}$ & 0.80300 & $0.79940[15]$ \\
& $308 \mathrm{~K}$ & 0.79880 & $0.79161[15]$ \\
& $313 \mathrm{~K}$ & 0.79632 & $0.78777[15]$ \\
\hline
\end{tabular}

\section{B. Methods}

The binary mixtures of 1-propanol and ethylene glycol of varied mole fractions were prepared by taking desired amount of ethylene glycol in $50 \mathrm{~cm} 3$ volumetric flak and adjusting the volume with required amount of 1- propanol. Values of different mole fractions of the binary mixtures were obtained with the help of density and volume data. Density of the pure liquids and binary mixtures have been recorded with the help of (DSA 5000M, Anton Paar). The operating frequency of the densitometer is $2 \mathrm{MHz}$ having accuracy of $\pm 0.00001 \mathrm{~g} / \mathrm{cm} 3$. The built in thermostat maintains the temperature up to $\pm 0.01 \mathrm{~K}$. Standardization has been carried out with distilled water. The dielectric constants were recorded with dipole meter (Mittal Enterprises, New Delhi). The dipole meter was calibrated with air and reference liquid. The required temperature has been maintained with the aid of digital constant temperature water bath which facilitates the circulation of water through the measuring cell which is double walled. $500 \mathrm{KHz}$ frequency is maintained by the circuit built audio oscillator present inside the equipment. The dipole meter holds an accuracy of \pm 0.005 . For measurement of dielectric permittivity of the binary mixtures maximum $50 \mathrm{~cm} 3$ for all the mole fractions has been used.

\section{THEORETICAL STUDIES}

The VE of the binary mixture was calculated with the help of the given equation:

$$
V^{E}=\sum_{i=1}^{N} X_{i} M_{i}\left(\rho^{-1}-\rho_{i}^{-1}\right)
$$

Where $\rho$ represents the density of the mixture, $\rho \mathrm{i}$ is the density of the pure component, $\mathrm{Xi}$ is the mole fraction and $\mathrm{N}$ indicates the number of unlike components.

The molar polarization $(\mathrm{P})$ of the binary mixture was obtained utilizing the equation given below:

$$
\mathrm{P}=\frac{(z-1)}{(z+2)} \cdot V
$$

Where $\varepsilon$ is the dielectric permittivity and $\mathrm{V}$ is the molar volume of the binary mixture.

The excess dielectric constant and molar polarization were calculated using

$$
A^{E}=\mathrm{A}-\left(A_{1} X_{1}+A_{2} X_{2}\right)
$$

$\mathrm{AE}$ represents the excess properties $(\varepsilon \mathrm{E}, \mathrm{PE}), \mathrm{A} 1$ and $\mathrm{A} 2$ represent the same parameters for individual components. Excess molar volume, ${ }^{E}\left(\mathrm{~cm}^{3} \cdot \mathrm{mol}^{-1}\right)$, dielectric permittivity, $\left(\varepsilon^{E}\right)$ and molar polarization, $P^{E}\left(\mathrm{~cm}^{3} \cdot \mathrm{mol}^{-1}\right)$ were fitted to Redlich- Kister polynomials of the form:

$$
V^{E}, \varepsilon^{E}, P^{E}=X_{1} X_{2} \sum_{i=0}^{m} A_{i}\left(2 X_{2}-1\right)^{i}
$$

Resolving a set of linear equations using MATLAB $\mathrm{R} 2015 \mathrm{~b}$, the coefficients $\mathrm{Ai}$ were obtained and the polynomial degree is represented as $\mathrm{m}$.

Using equation 5, the standard deviation $(\sigma)$ was calculated; $\mathrm{n}$ and $\mathrm{P}$ represents the data points and coefficients.

$$
\sigma(\mathrm{AE})=\left[\sum\left(A_{\theta x p}^{E}-A_{c a l}^{E}\right)^{2} /(n-p)\right]^{1 / 2}
$$




\section{RESULTS AND DISCUSSION}

Analysis of excess properties of liquid is important to study the interaction in binary mixtures [19]. The densities $(\rho)$, excess molar volume (VE), excess dielectric permittivity $(\varepsilon \mathrm{E})$ and excess molar polarization $(\mathrm{PE})$ on mixing ethylene glycol and 1- propanol at (298, 308 and 313 $\mathrm{K})$ are given in Table 2.

Table 2 Representation of density $\rho$, excess molar volume excess dielectric permittivity $\& E$ and excess molar polarization $\mathrm{PE}$ of binary mixture of ethylene

glycol and 1- propanol at 298, 308 and 313K.

\begin{tabular}{|c|c|c|c|c|}
\hline$X_{2}$ & $\rho\left(\mathrm{g} / \mathrm{cm}^{3}\right)$ & \multicolumn{2}{|c|}{$V^{E}\left(\mathrm{~cm}^{3} \cdot m \varepsilon^{E}\right.$} & \multirow[t]{2}{*}{$P^{E}$} \\
\hline \multicolumn{4}{|c|}{ Temp $=298 \mathrm{~K}$} & \\
\hline 0 & 0.80300 & 0 & 0 & 0 \\
\hline 0.178 & 0.84739 & -0.144 & -1.557 & -0.044 \\
\hline 0.278 & 0.87424 & -0.220 & -1.458 & 0.206 \\
\hline 0.403 & 0.91068 & -0.364 & -2.066 & 0.096 \\
\hline 0.519 & 0.94588 & -0.420 & -2.219 & 0.061 \\
\hline 0.654 & 0.99032 & -0.502 & -2.010 & -0.051 \\
\hline 0.700 & 1.00365 & -0.518 & -1.810 & -0.023 \\
\hline 0.811 & 1.04112 & -0.44 & -1.290 & -0.071 \\
\hline 0.981 & 1.10056 & -0.162 & -0.261 & -0.120 \\
\hline 1 & 1.10500 & 0 & 0 & 0 \\
\hline \multicolumn{5}{|c|}{ Temp $=308 \mathrm{~K}$} \\
\hline 0 & 0.7988 & 0 & 0 & 0 \\
\hline 0.178 & 0.84431 & -0.233 & -1.980 & -0.248 \\
\hline 0.278 & 0.87168 & -0.341 & -2.044 & -0.005 \\
\hline 0.403 & 0.90804 & -0.464 & -3.132 & -0.197 \\
\hline 0.519 & 0.94361 & -0.531 & -3.242 & -0.178 \\
\hline 0.654 & 0.98723 & -0.542 & -3.090 & -0.202 \\
\hline 0.700 & 1.00003 & -0.521 & -2.621 & -0.079 \\
\hline 0.811 & 1.03714 & -0.411 & -1.571 & -0.023 \\
\hline 0.981 & 1.09551 & -0.06 & -0.211 & -0.014 \\
\hline 1 & & & 0 & 0 \\
\hline \multicolumn{5}{|c|}{ Temp $=313 \mathrm{~K}$} \\
\hline 0 & 0.79632 & 0 & 0 & 0 \\
\hline 0.178 & 0.84283 & -0.335 & -2.583 & -0.666 \\
\hline 0.278 & 0.87011 & -0.441 & -2.605 & -0.333 \\
\hline 0.403 & 0.90864 & -0.728 & -3.466 & -0.574 \\
\hline 0.519 & 0.94316 & -0.717 & -3.900 & -0.549 \\
\hline 0.654 & 0.98655 & -0.713 & -3.580 & -0.477 \\
\hline 0.700 & 0.99964 & -0.711 & -3.211 & -0.370 \\
\hline 0.811 & 1.03851 & -0.700 & -2.011 & -0.351 \\
\hline 0.981 & 1.09311 & -0.141 & -0.233 & -0.091 \\
\hline 1 & 1.098 & 0 & 0 & 0 \\
\hline
\end{tabular}

The densities of the binary mixtures of ethylene glycol and 1-propanol were recorded at varied temperatures. The difference between the observed thermodynamic parameter of a mixture and the parameter for an ideal liquid is called as the excess function or property. Study of excess thermodynamic properties of liquid mixtures is vital to predict the nature of molecular interaction. The sign and magnitude of excess molar volume gives idea about the strength of molecular interaction. Negative VE indicate chemical interactions whereas positive VE results from unfavorable interactions.

\section{A. Excess molar volume}

Excess molar volumes represented in (Figure 2) and the values of VE follow a negative trend over the entire range of mole fraction at the measured temperature [20]. The main factors that affect the excess molar volume are the hydrogen bonding, molecular sizes and shapes. Alcohol molecules present in the binary mixture are strongly associated through hydrogen bonding. The values of VE are found to depend on temperature and become negative with increase in temperature $\mathrm{T}=(298-313) \mathrm{K}$. VE becomes more negative with rise in mole fraction of glycol, reaches a minimum then gradually increases at higher mole fraction. Intermolecular association at higher concentration of propanol is more whereas it decreases with increase in concentration of glycol. Attractive interactions between like molecules become weak with increase in temperature resulting in expansion of volume. The space between larger molecules becomes available for smaller molecules resulting in interstitial accommodation and efficient packing.

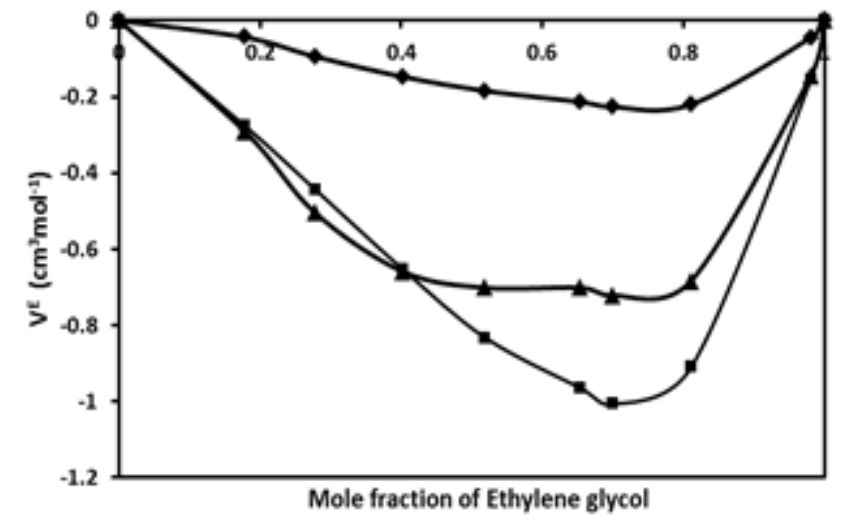

Figure 2: Variation of VE versus mole fraction of ethylene glycol for the binary system of ethylene glycol and 1-propanol at $\mathrm{T}=(-\uparrow-298 \mathrm{~K},-\square-308 \mathrm{~K}$ and $\Delta$ - 313 K).

\section{B. Permittivity and mixing rules}

Dielectric studies of the binary mixture of polar-nonpolar, polar-polar components are vital for understanding various inter and intra molecular interactions. In the present investigation, three mixing rules have been used for evaluation of dielectric permittivity suggested by Looyenga, Kraszewski and Lichtenecker-Rother [21]. Permittivity values of binary mixtures have been compared with the following mixing rules;

(i) Looyenga

$\varepsilon=\left[\varepsilon_{1}^{1 / 3}+\phi_{2}\left(\varepsilon_{2}^{\frac{1}{s}}-\varepsilon_{1}^{\frac{1}{3}}\right)\right]^{3}$

(ii) Kraszewski

$\varepsilon^{1 / 2}=\varphi_{1} \varepsilon_{1}^{1 / 2}+\varphi_{2} \varepsilon_{2}^{1 / 2}$

(iii) Lichtenecker-Rother

$\varepsilon=\varepsilon_{1}^{\varphi 1} \varepsilon_{2}^{\varphi 2}$

where $\varepsilon 1, \varepsilon 2,{ }_{1}^{\varphi_{1}} \varphi_{2}$, are dielectric constants and volume fractions of 1-propanol and ethylene glycol, respectively. 
(iv) The volume fractions, are calculated as,

$$
\varphi_{i}=X_{i} V_{i} /\left(\sum_{i=1}^{2} X_{i} V_{i}\right)
$$

Where, the ith component has molar volumeVi .The root mean square deviation (RMSD) values for the above stated mixing rules have been calculated with the help of equation 10.

$$
\text { (v) } \quad \operatorname{RMSD}=\left(\frac{1}{n \sum\left(\varepsilon_{e x p-} s_{c a l}\right)^{2}}\right)^{1 / 2}
$$

Where, $\mathrm{n}$ is the number of data points and exp represents the observed data and ecal signifies the calculated value obtained using the different mixing rules. The experimental values of dielectric constants measured at 298, 308 and $313 \mathrm{~K}$ are compared with the predicted values and are presented in Table 3.

Table 3 List of experimental and predicted values of dielectric permittivity (E) Looyenga, Kraszewski and

\begin{tabular}{|c|c|c|c|c|}
\hline $\mathrm{X}_{2}$ & $\varepsilon(\mathrm{Expt})$ & $\begin{array}{l}\varepsilon \\
\text { (Looyenga } \\
\text { ) }\end{array}$ & $\begin{array}{l}\varepsilon \\
\text { (Kraszewsk } \\
\text { i) }\end{array}$ & $\begin{array}{l}\text { ( } \varepsilon \quad \text { Kraszes }(\varepsilon \\
\varepsilon \\
\text { (Lichtenecker- } \\
\text { ) Rother Roth } \\
\text { Rother) }\end{array}$ \\
\hline \multicolumn{5}{|c|}{ Temp $=298 \mathrm{~K}$} \\
\hline 0 & 20.017 & 20.017 & 20.017 & 20.017 \\
\hline 0.178 & 21.820 & 22.308 & 22.890 & 22.215 \\
\hline 0.278 & 23.919 & 23.777 & 24.629 & 23.658 \\
\hline 0.403 & 25.812 & 25.839 & 26.967 & 25.789 \\
\hline 0.519 & 27.979 & 28.000 & 29.263 & 27.992 \\
\hline 0.654 & 30.889 & 30.881 & 32.153 & 31.054 \\
\hline 0.700 & 32.209 & 31.794 & 33.027 & 32.029 \\
\hline 0.811 & 34.950 & 34.564 & 35.543 & 34.863 \\
\hline 0.981 & 39.3799 & 39.470 & 39.631 & 39.746 \\
\hline 1 & 40.021 & 40.021 & 40.021 & 40.021 \\
\hline \multicolumn{5}{|c|}{ Temp $=308 \mathrm{~K}$} \\
\hline 0 & 18.750 & 18.750 & 18.750 & 18.750 \\
\hline 0.178 & 20.195 & 21.044 & 21.662 & 20.927 \\
\hline 0.278 & 22.161 & 22.521 & 23.425 & 22.362 \\
\hline 0.403 & 23.610 & 24.599 & 25.794 & 24.487 \\
\hline 0.519 & 25.856 & 26.785 & 28.122 & 26.701 \\
\hline 0.654 & 28.748 & 29.709 & 31.053 & 29.792 \\
\hline 0.700 & 30.339 & 30.638 & 31.939 & 30.782 \\
\hline 0.811 & 33.642 & 33.462 & 34.495 & 33.681 \\
\hline 0.981 & 38.453 & 38.485 & 38.650 & 38.740 \\
\hline 1 & 39.050 & 39.050 & 39.050 & 39.050 \\
\hline \multicolumn{5}{|c|}{ Temp $=313 \mathrm{~K}$} \\
\hline 0 & 18.000 & 18.000 & 18.000 & 18.000 \\
\hline 0.178 & 18.707 & 20.203 & 20.797 & 20.089 \\
\hline 0.278 & 20.635 & 21.622 & 22.491 & 21.466 \\
\hline 0.403 & 22.211 & 23.618 & 24.766 & 23.505 \\
\hline 0.519 & 24.040 & 25.717 & 27.003 & 25.630 \\
\hline 0.654 & 26.992 & 28.526 & 29.818 & 28.596 \\
\hline 0.700 & 28.438 & 29.419 & 30.669 & 29.546 \\
\hline 0.811 & 31.802 & 32.132 & 33.124 & 32.332 \\
\hline 0.981 & 36.896 & 36.956 & 37.115 & 37.197 \\
\hline 1 & 37.500 & 37.500 & 37.500 & 37.500 \\
\hline
\end{tabular}
Lichtenecker-Rother at 298, 308 and 313K. different theoretical mixture rules are presented in Table 4.

Table 4 RMSD values of different mixing rules for ethylene glycol + 1- propanol at 298, 308 and $313 \mathrm{~K}$.

\begin{tabular}{cccc}
\hline \multicolumn{3}{c}{ Dielectric permittivity $(\varepsilon)$} \\
\hline Mixing rule & \multicolumn{3}{c}{$308 \mathrm{~K}$} \\
Looyenga & $0.084 \mathrm{~K}$ & 0.013 & $313 \mathrm{~K}$ \\
\cline { 2 - 4 } Kraszewski & 0.007 & 0.002 & 0.004 \\
Lichtenecker- & 0.117 & 0.014 & 0.001 \\
Rother & & & 0.004 \\
& &
\end{tabular}

Dielectric permittivities of the mixtures of polar liquids are significant for understanding types of molecular interactions such as dipole-dipole, dipole-induced dipole and hydrogen bonding interactions. As a consequence, significant deviations are noted from the ideal ones and also the excess permittivity $(\varepsilon \mathrm{E})$ furnishes information about the associations present in the liquid mixture. It can be seen from the (Figure 3) that negative $\varepsilon \mathrm{E}$ values for ethylene glycol +1 -propanol binary mixture are negative recorded at 298, 308 and $313 \mathrm{~K}$. This indicates the presence of polar association between the components leading to the formation of multimers through hydrogen bonding resulting in reduction of effective dipole moment. The variation of $\varepsilon \mathrm{E}$ at various temperatures can be attributed to different power of hydrogen bond interactions and net alignments resulting from dipolar interactions in polar binary mixture [7].

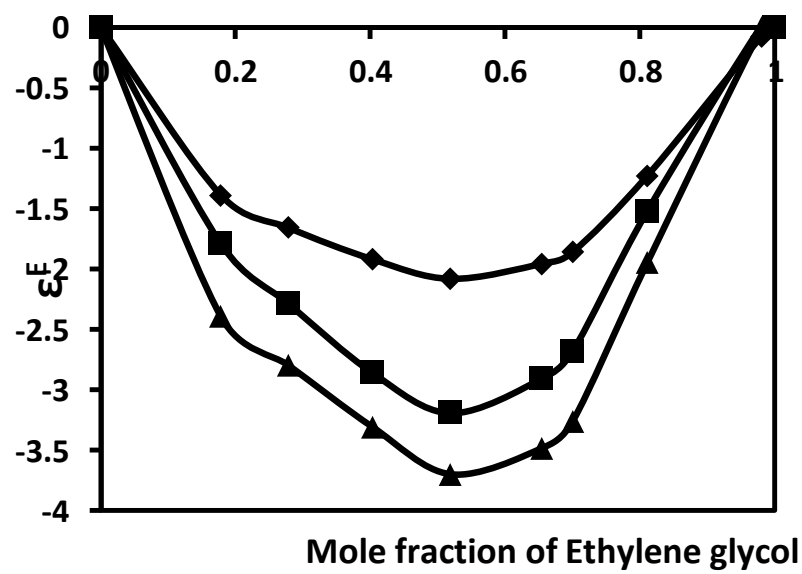

Figure 3: Variation of $\boldsymbol{\varepsilon E}$ versus mole fraction of ethylene glycol for the binary system of ethylene glycol and 1-propanol at $\mathrm{T}=(-\downarrow-298 \mathrm{~K},-\square-308 \mathrm{~K}$ and $-\Delta-313$ K).

\section{Excess molar polarization}

Values of excess molar polarization (PE) give good evidence about the interactions in the liquid mixture. Molar polarization provides information about the electrical properties of the molecule and is described by electrical properties per unit volume. PE identifies the range of 
interactions which can be short or long between like and unlike molecules. Positive PE indicates the associated region due to parallel alignment of molecular dipoles [22]. Figure 4 shows the variation of $\mathrm{PE}$ with different mole fractions of ethylene glycol. The shape of PE curves for $\mathrm{T}=$ 308 and $313 \mathrm{~K}$ are almost identical. But positive PE is observed at $298 \mathrm{~K}$ in 1- propanol rich region indicating selfassociation but with rise in mole fraction of ethylene glycol, it tends to become negative due to interaction between unlike components in the binary mixture. If the variations of $\mathrm{PE}$ in terms of temperature are considered, an increase of extrema is observed indicating favourable association between the unlike components. This may be due to geometrical fitting as a result of favourable association between like molecules which decreases with increase in temperature leading to expansion in volume. The coefficients (Ai) of the fitting for $\mathrm{VE}, \varepsilon \mathrm{E}$ and $\mathrm{PE}$ with their corresponding standard deviation $(\sigma)$ are listed in Table 5.

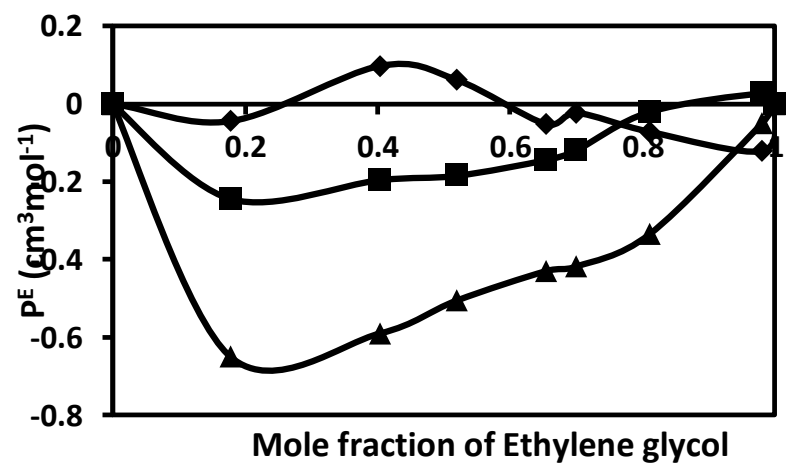

Figure 4: Variation of PE versus mole fraction of ethylene glycol for the binary system of ethylene glycol and 1-propanol at $\mathrm{T}=(-\downarrow-298 \mathrm{~K},--\mathbf{-}-308 \mathrm{~K}$ and $-\Delta-313$ K).

Table 5 Calculated values of Redlich Kister coefficients and standard deviations for the excess functions for ethylene glycol + 1- propanol.

\begin{tabular}{ccccccc}
$\begin{array}{c}\text { Excess } \\
\text { properti } \\
\text { es }\end{array}$ & $\begin{array}{c}\mathrm{Temp} \\
(\mathrm{K})\end{array}$ & $\mathrm{A}_{0}$ & $\mathrm{~A}_{1}$ & $\mathrm{~A}_{2}$ & $\mathrm{~A}_{3}$ & $\sigma$ \\
\hline$V^{E}$ & 298 & -0.7306 & -0.5916 & -0.3869 & -0.7779 & 0.0298 \\
& 308 & -3.2885 & -3.0062 & -1.6824 & -0.5292 & 0.0676 \\
& 313 & -2.8513 & -0.3973 & -1.0888 & -3.8848 & 0.0009 \\
$\varepsilon^{E}$ & 298 & -8.4271 & -2.0946 & -0.7144 & 8.3391 & 0.0129 \\
& 308 & - & -3.9075 & 4.8125 & 14.9175 & 0.0220 \\
& & 12.9125 & & & & \\
& 313 & - & -5.4196 & 1.3281 & 21.2291 & 0.0174 \\
$P^{E}$ & 298 & 0.3967 & -1.4961 & -1.2188 & 2.7544 & 0.0024 \\
& 308 & -0.7613 & 0.1858 & -0.2787 & 2.5883 & 0.0006 \\
& 313 & -2.1156 & 1.3374 & -2.9869 & 1.0611 & 0.0008 \\
& & & & & & \\
\hline
\end{tabular}

\section{CONCLUSIONS}

From the experimental densities, molar volumes, dielectric permittivity values, excess molar volumes, dielectric constants and molar polarizations were calculated at $\mathrm{T}=298,308$ and $313 \mathrm{~K}$. Negative deviations were observed in all the excess properties mostly over higher composition range of ethylene glycol at $\mathrm{T}=298,308$ and $313 \mathrm{~K}$ and this could be attributed to the presence of intermolecular interactions in the system studied. Out of the three mixing rules used for theoretical calculation of dielectric permittivity, Kraszewski model worked best. Redlich-Kister polynomial equation was used successfully for the correlation of VE, $\varepsilon E$ and PE. The basic findings of this work can be a source of supporting information regarding physicochemical behaviour of ethylene glycol and 1- propanol binary mixture where ethylene glycol is one of the active components used in extractive distillation due to its low toxicity and reduced vapour pressure.

\section{ACKNOWLEDGEMENT}

The authors express their gratefulness to authorities of Siksha 'O' Anusandhan Deemed to be University for giving opportunity to carry out this research work.

\section{REFERENCES}

1. C. Yang, W. Xu, P. Ma, "Thermodynamic properties of binary mixtures of p-xylene with cyclohexane, heptane, octane and N-methyl-2-pyrrolidone at several temperatures" in Journal of Chemical Engineering and Data, vol. 49, 2004, pp - $1794-1801$.

2. A. Pal, S. Sharma, "Excess molar volumes and viscosities of binary liquid mixtures of ethylene glycol dimethyl ether + ethylene glycol monomethyl, + diethylene glycol monomethyl, and + triethylene glycol monomethyl ethers at 298.15 and $308.15 \mathrm{~K}$ " in Journal of Chemical Engineering and Data, vol. 44, 1999, pp - 212 215.

3. F. Koohyar, F. Kiani, S. Sharifi, M. Sharifirad, S. H. Rahmanpour, "Study on the Change of Refractive Index on Mixing, Excess Molar Volume, and Viscosity Deviation for Aqueous Solution of Methanol, Ethanol, Ethylene Glycol, 1-Propanol, and 1, 2, 3-Propanetriol at $\mathrm{T}=292.15 \mathrm{~K}$ " in Research Journal of Applied Sciences, Engineering and Technology, vol. 4, 2012, pp- 3095 3101.

4. M. S. Cruz, L. D. A. Chumpitaz, J. G. L. F. Alves, A. J. A. Meirelles, "Kinematic viscosities of poly(ethylene glycols)"in Journal of Chemical Engineering and Data, vol. 45, 2000, pp - $61-63$.

5. C. Yang, P. Ma, F. Jing, D. Tang, "Excess molar volumes, viscosities and heat capacities for the mixtures of ethylene glycol + water from $273.15 \mathrm{~K}$ to $353.15 \mathrm{~K}$ " in Journal of Chemical Engineering Data, vol . 48, 2003, pp - 836 - 840.

6. T. P. Iglesias, J. P. Fernandez, "A new approach for prediction of the permittivity of mixtures" in Journal of Chemical Thermodynamics, vol. 33, 2001, pp -1375 1381.

7. S. K. Ray, B. Mohanty, S. Tripathy, G. S. Roy, "Evaluation of excess Gibbs energy of mixing in the binary mixtures tri-n-butyl phosphate in nonpolar solvents using dielectric probe" in Indian Journal of Pure and Applied Physics, vol. 37, 1999, pp - 127-132. 
8. H. A. Chaube, V. A. Rana, "Dielectric studies of binary mixtures ethylene glycol mono phenyl ether with 1butanol at different temperatures" in Journal of Advanced Dielectrics, vol. 7, 2017, pp - 1750023 (1-7).

9. V. A. Rana, H. A. Chaube, D. H. Gadani, "Dielectric permittivity, density, viscosity and refractive index of binary mixtures of anisole with methanoland 1- propanol at different temperatures" in Journal of Molecular Liquids, vol. 164, 2011, pp - 191-196.

10. M. T. Holtzapple, R. Davison, M. K. Ross, S. AldrettLee, M. Nagwani, C. M. Lee, M. E. Loescher, "Twentieth Symposium on Biotechnology for Fuels and Chemicals, Humana Press, New York", 1999, pp - 609631.

11. S. Atsumi, J. C. Liao, "Directed evolution of methanococcus jannaschii citramalate synthase for biosynthesis of 1- propanol and 1- butanol by Escherichia coli" in Applied and Environmental Microbiology, vol. 74, 2008, pp - $7802-7808$.

12. L. Albright, in Albright's Chemical Engineering Handbook, CRC Press, Bocaraton, 2008.

13. J. Pla-Franco, E. Lladosa, S. Loras, J. B. Monton, "Approach to the 1- propanol dehydration using an extractive distillation process with ethylene glycol" in Chemical Engineering and Processing, 2015, pp - 1-20.

14. C. Yang, Z. Liu, H. Lai, P. Ma, "Excess molar volumes and viscosities of binary mixtures of $p$ - cresol with ethylene glycol and methanol at different temperature and atmospheric pressure" in Journal of Chemical Engineering and Data, vol. 51, 2006, pp - 457 - 461.

15. S. M. Contreras, "Densities and viscosities of binary mixtures of 1, 4- dioxane with 1- propanol and 2propanol at $(25,30,35 \text { and } 40)^{\circ} \mathrm{C}$ " in Journal of Chemical Engineering and Data, vol. 46, 2001, pp $1149-1152$.

16. R. J. Sengwa, "A comparative dielectric study of ethylene glycol and propylene glycol at different temperatures" in Journal of Molecular Liquids, vol. 108, 2003, pp $-47-60$.

17. B. Lone, V. Madhurima, "Dielectric and conformal studies of 1- propanol and 1- butanol in methanol" in Journal of Molecular Modeling, vol.17, 2011, pp - 709 719.

18. A. D. Vyas, V. Rana, S. P .Bhatnagar, V. M. Vashisth, "Dielectric dispersion and relaxation of mixtures of 1 propanol and phenol at lower microwave frequencies" Indian Journal of Pure.and Applied Physics, vol. 49, 2011, pp - 277-283.

19. M. V. Rathnam, P. K. R. Singh, M. S. Kumar, "Excess volumes of binary solutions of methyl formate, ethyl formate, propyl formate and benzyl acetate with bromo-, chloro- and nitrobenzenes at (303.15, 308.15 and 313.15) K", in Journal of Chemical Engineering and Data, vol. 53, 2008, pp - $265-270$.

20. N. G. Tsierkezos, I. E. Molino, "Thermodynamic properties of water + ethylene glycol at 283.15, 293.15 and $313.15 \mathrm{~K}$ " in Journal of Chemical Engineering and Data, vol. 43, 1998, pp -989-993.

21. A. G. Gilani, H. G. Gilani, M. Ansari, "A thermodynamic study of solute solvent interactions through dielectric properties of the mixtures consisting of 1, 4-butanediol, 1-octanol, and 1, 4-dioxane at different temperatures", in Journal of chemical Thermodynamics, vol. 55, 2012, pp - $203-212$.

22. S. P. Kamble, Y. S. Sudake, S.S. Patil, P. W. Khirade, S. S. Mehrotra, "Dielectric and optical properties of polar and non-polar binary liquids at different temperature" in International Journal of Pharma and Bio Sciences, vol. 2, 2011, pp - $297-306$ 